\title{
Positionspapier zur Detektion von Vorhofflimmern nach ischämischem Schlaganfall
}

\author{
Arbeitsgemeinschaft Herz und Hirn der Deutschen Gesellschaft \\ für Kardiologie - Herz- und Kreislaufforschung e.V. (DGK) und der \\ Deutschen Schlaganfall-Gesellschaft e.V. (DSG)
}

\section{Position Paper on Atrial Fibrillation Detection After Ischemic Stroke}

\author{
Working Group Heart and Brain of the German Cardiac Society (DGK) and \\ the German Stroke Society (DSG)
}

\begin{abstract}
Autoren
Karl Georg Häusler ${ }^{1,2,3}$, Klaus Gröschel ${ }^{4}$, Martin Köhrmann ${ }^{5}$, Renate B. Schnabel 3,6,7, Stefan D. Anker ${ }^{8,9}$, Johannes Brachmann ${ }^{10}$, Michael Böhm ${ }^{11}$, Hans-Christoph Diener ${ }^{5}$, Wolfram Doehner ${ }^{2,12}$, Matthias Endres 1,2, 13, 14, 15, Christian Gerloff ${ }^{16,17}$, Hagen B. Huttner ${ }^{18}$, Manfred Kaps ${ }^{19}$, Paulus Kirchhof ${ }^{3,20}$, Darius Günther Nabavi ${ }^{17,21}$, Christian H. Nolte 1,2,13, Waltraud Pfeilschifter ${ }^{22}$, Burkert Pieske ${ }^{12,14,23}$, Sven Poli ${ }^{24}$, Wolf Rüdiger Schäbitz ${ }^{25}$, Götz Thomalla ${ }^{16}$, Roland Veltkamp ${ }^{26}$, Thorsten Steiner ${ }^{27,28}$, Ulrich Laufs ${ }^{11,29}$, Joachim Röther ${ }^{30}$, Rolf Wachter ${ }^{29,31}$
\end{abstract}

Institute

1 Klinik und Hochschulambulanz für Neurologie, Charité - Universitätsmedizin Berlin

2 Centrum für Schlaganfallforschung Berlin, Charité Universitätsmedizin Berlin

3 Kompetenznetz Vorhofflimmern e.V.

4 Klinik und Poliklinik für Neurologie, Universitätsmedizin Mainz, Mainz

5 Klinik für Neurologie, Universitätsklinikum Essen, Essen

6 Abteilung für Allgemeine und Interventionelle Kardiologie, Universitäres Herzzentrum Hamburg

7 Deutsches Zentrum für Herz-Kreislauf-Forschung e.V. (DZHK), Standort Hamburg/Kiel/Lübeck

8 Abteilung Kardiologie und Stoffwechsel - Herzinsuffizienz, Kachexie \& Sarcopenie, Medizinische Klinik mit Schwerpunkt Kardiologie, Campus Virchow-Klinikum, Charité - Universitätsmedizin Berlin; Berlin-Brandenburg Center for Regenerative Therapies (BCRT), Charité - Universitätsmedizin Berlin

9 Klinik für Kardiologie und Pneumologie, Universitätsmedizin Göttingen (UMG) \& Deutsches Zentrum für Kardiovaskuläre Forschung (DZHK), Standort Berlin

10 Medizinische Klinik für Innere Medizin und Kardiologie, Klinikum Coburg

11 Klinik für Innere Medizin III, Kardiologie, Angiologie und internistische Intensivmedizin, Universitätsklinikum des Saarlandes, Homburg

12 Klinik für Innere Medizin mit Schwerpunkt Kardiologie, Campus Virchow Klinikum, Berlin, Charité - Universitätsmedizin Berlin

13 Berlin Institute of Health
14 Deutsches Zentrum für Herz-Kreislauf-Forschung e.V. (DZHK), Standort Berlin

15 Deutsches Zentrum für Degenerative Erkrankungen (DZNE), Standort Berlin

16 Klinik und Poliklinik für Neurologie, Kopf- und Neurozentrum, Universitätsklinikum Eppendorf, Hamburg

17 Stroke Unit Kommission der Deutschen SchlaganfallGesellschaft

18 Neurologische Klinik, Universitätsklinikum Erlangen, Erlangen

19 Klinik und Poliklinik für Neurologie, Universitätsklinikum Gießen

20 Institute of Cardiovascular Sciences, University of Birmingham, Sandwell and West Birmingham Hospitals NHS trust, University Hospitals Birmingham NHS Foundation NHS trust, Birmingham, Großbritannien

21 Klinik für Neurologie, Vivantes Klinikum Neukölln, Berlin

22 Zentrum der Neurologie und Neurochirurgie, Klinik für Neurologie, Universitätsklinikum Frankfurt

23 Klinik für Innere Medizin und Kardiologie, Deutsches Herzzentrum Berlin

24 Abteilung Neurologie mit Schwerpunkt neurovaskuläre Erkrankungen und Hertie-Institut für klinische Hirnforschung, Universitätsklinikum Tübingen, Tübingen

25 Klinik für Neurologie, Evangelisches Klinikum Bethel, Bielefeld

26 Department of Stroke Medicine, Imperial College London, Großbritannien

27 Neurologische Klinik, Klinikum Frankfurt Höchst, Frankfurt 
28 Neurologische Klinik, Universitätsklinik Heidelberg, Heidelberg

29 Klinik und Poliklinik für Kardiologie, Universitätsklinikum Leipzig, Leipzig

30 Abteilung für Neurologie, Asklepios Klinik Altona, Hamburg

31 Klinik für Kardiologie und Pneumologie, Universitätsmedizin Göttingen \& Deutsches Zentrum HerzKreislauf-Forschung e.V., Standort Göttingen

Schlüsselwörter

ischämischer Schlaganfall, Vorhofflimmern,

EKG-Monitoring

Keywords

ischemic stroke, atrial fibrillation, ECG monitoring

Bibliografie

DOI https://doi.org/10.1055/s-0043-118476

Online-Publikation: 26.10.2017 | Akt Neurol 2018; 45:

93-106

(c) Georg Thieme Verlag KG Stuttgart · New York

ISSN 0302-4350

Korrespondenzadresse

Priv.-Doz. Dr. med. Karl Georg Häusler, Klinik und

Hochschulambulanz für Neurologie, Charité -

Universitätsmedizin Berlin, Campus Benjamin Franklin,

Hindenburgdamm 30,

12203 Berlin

georg.haeusler@charite.de

\section{ZUSAMMENFASSUNG}

Das vorliegende Positionspapier zur Detektion von Vorhofflimmern nach ischämischem Schlaganfall beinhaltet eine Stellungnahme der Arbeitsgemeinschaft „Herz und Hirn“ der Deutschen Gesellschaft für Kardiologie - Herz- und Kreislaufforschung e. V. (DGK) und der Deutschen Schlaganfallgesellschaft e. V. (DSG), die in Zusammenarbeit mit der Stroke Unit-Kommission der DSG und dem Kompetenznetz Vorhofflimmern (AFNET) e. V. erstellt wurde.

Vorhofflimmern ist in den Leitlinien der Europäischen Gesellschaft für Kardiologie als eine mindestens 30 Sekunden anhaltende Episode einer Vorhofarrhythmie mit fehlenden P-Wellen definiert. Die 30-Sekundengrenze ist arbiträr gewählt und es ist unbekannt, ob das Schlaganfallrisiko bei Episoden von länger als 30 Sekunden höher ist als bei kürzeren Episoden.

Bei Patienten, die einen Schlaganfall erlitten haben, sollte die Detektion von Vorhofflimmern üblicherweise zu einer Umstellung der medikamentösen Sekundärprävention führen, da eine orale Antikoagulation einer Thrombozytenaggregationshemmung überlegen ist. Die Detektion eines bis dato nicht diagnostizierten Vorhofflimmerns sollte daher bei Patienten mit ischämischem Schlaganfall verbessert werden, um die medikamentöse Sekundärprävention des Schlaganfalls zu optimieren.

In diesem Positionspapier werden interdisziplinär erarbeitete Standards für eine „strukturierte Rhythmusvisite“ auf der Stroke Unit und ein diagnostisches „Stufenschema“ zur Detektion von Vorhofflimmern vorgeschlagen. Das Positionspapier fasst zudem den gegenwärtigen Kenntnisstand zur Detektion von Vorhofflimmern nach ischämischem Schlaganfall zusammen. Das Positionspapier soll den in der Versorgung von Schlaganfallpatienten tätigen Ärzten eine Handhabe geben, auch wenn bisher nicht abschließend geklärt ist, wie lange und mit welcher EKG-Methode Schlaganfallpatienten ohne bisher bekannte Herzrhythmusstörung bestmöglich monitoriert werden sollten. Dem Charakter eines Positionspapiers angemessen, wurde auf die Kennzeichnung von Evidenzgraden verzichtet, da es sich überwiegend um die Meinung von Experten handelt, die auf berichteten Fallserien und klinischer Erfahrung beruht und somit nicht mit einer Leitlinie gleichzusetzen ist und so auch nicht verstanden werden will.

\section{ABSTRACT}

This position paper on the detection of atrial fibrillation after ischemic stroke includes a statement of the "Heart and Brain" consortium of the German Cardiac Society and the German Stroke Society. This paper was endorsed by the Stroke Unit-Commission of the German Stroke Society and the German Atrial Fibrillation NETwork.

The guidelines of the European Cardiac Society define atrial fibrillation as atrial arrhythmia lasting at least 30 seconds with missing P-waves. The limit of 30 seconds was chosen arbitrarily, and it is unknown whether the risk of ischemic stroke is higher for episodes lasting longer than 30 seconds compared to shorter episodes.

In patients with ischemic stroke, detection of atrial fibrillation should usually lead to a change in secondary stroke prevention since oral anticoagulation is superior to antiplatelet drugs. The detection of previously undiagnosed atrial fibrillation should therefore be improved in patients with ischemic stroke in order to optimize medical stroke prevention. This position paper summarizes the present knowledge on the detection of atrial fibrillation after ischemic stroke. An interdisciplinary standard for a "structured analysis of ECG monitoring" on the Stroke Unit is proposed as well as a diagnostic "level scheme for the detection of atrial fibrillation". In light of the fact that the optimal duration and mode of ECG monitoring of patients with so far unknown cardiac arrhythmia has not yet been finally established, this position paper is intended to give advice to physicians who are involved in stroke care. In line with the nature of a position paper, the labeling of classes of recommendations is not provided, since many statements are based on the opinion of experts, reported case series and clinical experience. Therefore, this position paper is not intended as a guideline. 


\section{Einleitung}

Der Schlaganfall ist die vierthäufigste Todesursache und die führende Ursache für erworbene Behinderungen in Deutschland. Allein in Deutschland treten pro Jahr etwa 260000 Schlaganfälle auf [1,2]. Etwa 15-20\% dieser Schlaganfälle werden durch Vorhofflimmern bedingt, das derzeit etwa $2 \%$ aller Bundesbürger betrifft und das Risiko für einen ischämischen Schlaganfall im Vergleich zur Allgemeinbevölkerung etwa vierbis fünffach erhöht $[3,4]$. Schlaganfallpatienten mit Vorhofflimmern weisen zudem aufgrund eines vergleichsweise hohen Rezidiv-Risikos und einer erhöhten Sterblichkeit eine schlechtere Prognose auf als Schlaganfallpatienten ohne Vorhofflimmern [5], was den Stellenwert der Schlaganfallprävention unterstreicht. Vorhofflimmern tritt bei einem relevanten Anteil der Betroffenen jedoch nur intermittierend auf und verläuft häufig asymptomatisch, was auch für die Mehrzahl der Schlaganfallpatienten zutrifft, bei denen im weiteren Verlauf erstmals ein Vorhofflimmern detektiert wird [6]. Die Detektion von Vorhofflimmern nach ischämischem Schlaganfall ist aus folgenden Gründen relevant:

- Schlaganfallpatienten mit (unentdecktem) Vorhofflimmern haben unbehandelt ein vergleichsweise hohes Rezidivrisiko für einen ischämischen Schlaganfall [5,7].

- Schlaganfälle, die bestimmte Hirnareale (wie beispielsweise die Inselregion) betreffen, können möglicherweise das Auftreten von Vorhofflimmern begünstigen $[8,9]$.

- Ein in der Akutphase des Schlaganfalls erstmals nachgewiesenes Vorhofflimmern ist bei der überwiegenden Mehrzahl der Betroffenen auch im Verlauf nachweisbar [10].

- Der Nachweis von Vorhofflimmern ist für die medikamentöse Sekundärprävention des ischämischen Schlaganfalls relevant [4], auch wenn ein nachgewiesenes Vorhofflimmern nicht immer gleichbedeutend mit einer kardio-embolischen Genese des Schlaganfalls ist [11-13]. Orale Antikoagulanzien sind bei fehlenden Kontraindikationen in der Sekundärprävention des ischämischen Schlaganfalls bei bestehendem Vorhofflimmern effektiv und erreichen im Vergleich zu Placebo eine relative Risikoreduktion von $70-80 \%[14,15]$. Patienten mit einer erstmaligen Vorhofflimmer-Episode nach einem akuten ischämischen Schlaganfall waren in den für die Zulassung der oralen Antikoagulanzien relevanten Phase-III-Studien jedoch unterrepräsentiert.

- Eine bei Auftreten eines ischämischen Schlaganfalls therapeutisch wirksame orale Antikoagulation ist mit einer Reduktion des Schlaganfall-Schweregrades assoziiert $[16,17]$.

\section{Aktuelle Leitlinien und Empfehlungen}

Bei Schlaganfallpatienten korreliert die Dauer des EKG-Monitorings [18, 19], die Qualität der Auswertung [20,21] und die Patientenselektion [22,23] mit der Häufigkeit von detektiertem Vorhofflimmern. In den letzten Jahren wurden zudem klinische, laborchemische, echokardiografische oder elektrokardiografische Parameter identifiziert, die im Rahmen eines verlängerten EKG-Monitorings nach ischämischem Schlaganfall mit einer er- höhten Wahrscheinlichkeit für einen erstmaligen Nachweis von Vorhofflimmern einhergehen.

Für Patienten mit ischämischem Schlaganfall wurde im Jahr 2010 in einem Positionspapier der AG Herz \& Hirn [24] ein EKG-Monitoring von zumindest 24 Stunden empfohlen. In den Leitlinien der Deutschen Gesellschaft für Neurologie (DGN) $[25,26]$ und der European Stroke Organisation (ESO) [27] wird neben der Ableitung eines 12-Kanal-Ruhe-EKGs bei Aufnahme und dem kontinuierlichen EKG-Monitoring auf der Stroke Unit eine Langzeit-EKG-Registrierung bei Schlaganfallpatienten mit fortbestehendem dringendem Verdacht auf ein asymptomatisches, paroxysmales Vorhofflimmern empfohlen, ohne dass konkrete Angaben zur Dauer des EKG-Monitorings in der Akutphase gemacht werden. Die US-amerikanischen Leitlinien empfehlen seit der letzten Aktualisierung im Jahr 2014 für Patienten mit kryptogenem Schlaganfall innerhalb der letzten 6 Monate ein EKG-Monitoring von 30 Tagen (Klasse Ila, Level C) [28]. Die 2016 publizierten Leitlinien der Europäischen Gesellschaft für Kardiologie zur Therapie des Vorhofflimmerns empfehlen für Schlaganfallpatienten und Patienten mit einer transitorisch ischämischen Attacke (TIA) ohne bekanntes Vorhofflimmern ein EKG-Monitoring von zumindest 72 Stunden zur Detektion eines bis dato nicht erkannten Vorhofflimmerns (Klasse I, Level B) $[4]$.

Auch die zuletzt im Jahr 2015 aktualisierten Zertifizierungskriterien der DSG empfehlen neben einem EKG-Monitoring mit einer zentralen Überwachung eine ausreichende Verfügbarkeit von Langzeit-EKGs im Regeldienst sowie einen schriftlich festgelegten Standard zum Einsatz des Langzeit-EKGs. Im Rahmen der letzten Überarbeitung der Zertifizierungskriterien wurde das Kriterium „Detektion von Vorhofflimmern“ hinzugefügt, das ein „erweitertes Diagnostikkonzept für Patienten mit kryptogenem Insult“ empfiehlt, dies jedoch nicht als Minimalanforderung für die Zertifizierung definiert [29]. Neben strukturierten Rhythmusvisiten und der Nutzung einer speziellen Detektionssoftware für Monitorsysteme wird auf die Implantation von Ereignisrekordern hingewiesen.

\section{Studienlage zur Detektion von Vorhofflimmern nach ischämischem Schlaganfall}

Bisher sind die Ergebnisse von vier randomisierten Studien zur Effizienz eines verlängerten EKG-Monitorings bei Patienten mit akutem bzw. bis dato als kryptogen klassifiziertem ischämischem Schlaganfall vorgestellt worden ( Tab. 1) [30-33]. Diese Studien haben anhand unterschiedlicher Monitoring-Verfahren nachgewiesen, dass diese die Detektionsrate von Vorhofflimmern erhöhen und die Anzahl der konsekutiv antikoagulierten Schlaganfallpatienten gesteigert werden kann. Die Vorhofflimmerdetektionsrate korrelierte dabei mit der Dauer des EKGMonitorings, wobei die Detektionsrate im Verlauf des Monitorings abnahm [33].

Die Patientenzahl bzw. die Dauer der Nachverfolgung dieser randomisierten Studien waren jedoch nicht darauf ausgelegt belegen zu können, dass ein verlängertes EKG-Monitoring eine 
- Tab. 1 Vergleich der Basischarakteristika und Ergebnisse von vier randomisierten Studien zum verlängerten EKG-Monitoring nach ischämischem Schlaganfall.

\begin{tabular}{|c|c|c|c|c|c|c|}
\hline $\begin{array}{l}\text { Studie } \\
\text { [Referenz] }\end{array}$ & $\begin{array}{l}\text { Monitoring- } \\
\text { Verfahren }\end{array}$ & $\mathbf{n}$ & $\begin{array}{l}\text { Alter } \\
\text { (Jahre) } \\
\text { [Min.] }\end{array}$ & $\begin{array}{l}\text { Einschluss- } \\
\text { fenster }\end{array}$ & $\begin{array}{l}\text { Vorhofflimmer- } \\
\text { nachweis (Number } \\
\text { needed to screen) }\end{array}$ & Kommentar \\
\hline $\begin{array}{l}\text { Higgins et al. } \\
{[30]}\end{array}$ & 7 Tage Holter EKG & 100 & $\begin{array}{l}66 \pm 12 \\
{[\geq 18]}\end{array}$ & $<7$ Tage & $\begin{array}{l}18 \% \text { vs. } 2 \% \text { nach } \\
14 \text { Tagen }\end{array}$ & $\begin{array}{l}\text { Pilotstudie für eine geplante } \\
\text { Studie mit bis zu } 5000 \\
\text { Patienten }\end{array}$ \\
\hline $\begin{array}{l}\text { EMBRACE } \\
{[32]}\end{array}$ & $\begin{array}{l}\text { 30-Tage Ereignis- } \\
\text { rekorder vs. } \\
24 \text { Stunden Holter } \\
\text { EKG }\end{array}$ & 572 & $\begin{array}{l}73 \pm 9 \\
{[\geq 55]}\end{array}$ & <6 Monate & $\begin{array}{l}16,1 \% \text { vs. } 3,2 \% \text { nach } \\
30 \text { Tagen }\end{array}$ & $\begin{array}{l}\text { abnehmende Compliance } \\
\text { mit Dauer des Monitorings. } \\
\text { Über mindestens } 3 \text { Wochen } \\
\text { wurden } 82 \% \text { monitoriert. }\end{array}$ \\
\hline $\begin{array}{l}\text { CRYSTAL-AF } \\
\text { [31] }\end{array}$ & $\begin{array}{l}\text { implantierbarer } \\
\text { Ereignisrekorder vs. } \\
\text { „Standard“ }\end{array}$ & 441 & $\begin{array}{l}61 \pm 11 \\
{[\geq 40]}\end{array}$ & $<3$ Monate & $\begin{array}{l}8,9 \% \text { vs. } 1,4 \% \text { nach } \\
180 \text { Tagen (14) } \\
12,4 \% \text { vs. } 2,0 \% \text { nach } \\
1 \text { Jahr (10) } \\
30,0 \% \text { vs. } 3,0 \% \text { nach } \\
3 \text { Jahren (4) }\end{array}$ & $\begin{array}{l}\text { „Standard“-Gruppe: } \\
\text { Nur etwa ein Drittel aller } \\
\text { Studienpatienten erhielt } \\
\text { weitere EKG-Ableitung in } \\
180 \text { Tagen. }\end{array}$ \\
\hline $\begin{array}{l}\text { FIND AF randomised } \\
\text { [33] }\end{array}$ & $\begin{array}{l}\text { 10-Tage EKG bei } \\
\text { Aufnahme, nach } \\
3 \text { \& } 6 \text { Monaten vs. } \\
\text { „Standard“ }\end{array}$ & 402 & $\begin{array}{l}73 \pm 7 \\
{[\geq 60]}\end{array}$ & $\leq 7$ Tage & $\begin{array}{l}13,5 \% \text { vs. } 4,5 \% \text { nach } \\
180 \text { Tagen (11) } \\
13,5 \% \text { vs. } 6,1 \% \text { nach } \\
1 \text { Jahr (14) }\end{array}$ & $\begin{array}{l}\text { Die Teilnahme am 1., 2., } 3 . \\
\text { 10-Tage-Langzeit-EKG } \\
\text { betrug } 100 \%, 68 \% \text { bzw. } 65 \% \text {. }\end{array}$ \\
\hline
\end{tabular}

Reduktion klinischer Endpunkte nach sich zieht. Die Relevanz eines verlängerten stationären nicht-invasiven EKG-Monitorings nach akutem ischämischem Schlaganfall oder TIA wird in Deutschland derzeit im Rahmen der multizentrischen randomisierten MonDAFIS-Studie untersucht. Bis August 2017 sollen insgesamt 3470 Schlaganfallpatienten in die MonDAFIS-Studie eingeschlossen werden, deren primärer Endpunkt die Antikoagulationsrate nach einem Jahr ist. Die Häufigkeit von Schlaganfällen, Myokardinfarkten, schweren Blutungen und Todesfällen ist ein prädefinierter sekundärer Endpunkt der Studie [34].

Mehrere prospektive Kohortenstudien (zur Übersicht [34]) konnten belegen, dass die Detektion von Vorhofflimmern nach ischämischem Schlaganfall nicht nur von der Dauer des Monitorings sondern auch vom Zeitpunkt des EKG-Monitorings in Relation zum Index-Schlaganfall und von der Patientenauswahl abhängig ist ( $\triangleright$ Tab. 2). Aufgrund der Heterogenität der Patientenkohorten, der eingesetzten EKG-Methoden, der verwendeten Vorhofflimmerdefinition und der Ableitungsdauer sind publizierte Metaanalysen nur bedingt aussagekräftig [18], lassen jedoch den Rückschluss zu, dass die Nachweisrate von Vorhofflimmern in selektierten Schlaganfallkohorten höher ist als in unselektierten Schlaganfallkohorten [22].

\section{Definition von Vorhofflimmern und Relevanz der Vorhofflimmer-Typisierung}

Nach Expertenmeinung erfordert die Diagnose „Vorhofflimmern" eine mindestens 30 Sekunden anhaltende Episode einer Vorhofarrhythmie mit fehlenden P-Wellen. Kürzere Episoden (<30 Sekunden) sollten nicht als Vorhofflimmern, sondern als „atrial run“ oder vermehrte supraventrikuläre Ektopie benannt werden. Die 30-Sekundengrenze wird auch in den geltenden
Leitlinien der Europäischen Gesellschaft für Kardiologie erwähnt [4], wobei diese Definition nicht prospektiv evaluiert wurde und auf Konvention sowie auf Einschlusskriterien von klinischen Studien beruht. Bei bis zu $44 \%$ aller Patienten mit einem akuten ischämischen Schlaganfall finden sich in der Akutphase „atriale runs“ $[30,36]$, deren prognostische Relevanz nicht abschließend geklärt ist.

Die derzeitige Datenlage legt nach Ansicht der Autoren den Schluss nahe, dass die Dauer von Vorhofflimmer-Episoden für das Schlaganfallrisiko eine eher untergeordnete Relevanz besitzt [37, 38]. Eine Metaanalyse prospektiver Studien zur (Primär-)Prävention des ischämischen Schlaganfalls postulierte jedoch ein etwas höheres Schlaganfallrisiko für Patienten mit persistierendem oder permanentem Vorhofflimmern im Vergleich zu Patienten mit paroxysmalem Vorhofflimmern [39]. Eine retrospektive Analyse der ASSERT-Studie legt zudem den Schluss nahe, dass bei Schrittmacherpatienten das Schlaganfallrisiko erst ansteigt, wenn eine atriale Tachykardie zumindest einmalig über mindestens 24 Stunden anhielt [40]. Eine Korrelation des Vorhofflimmer-Typs mit dem Schlaganfallrisiko ist für die Sekundärprävention des Schlaganfalls bisher nicht belegt und sollte für therapeutische Entscheidungen derzeit keine Berücksichtigung finden, zumal die klinische Klassifikation des Vorhofflimmer-Typs bei einem relevanten Anteil von Patienten nicht korrekt erfolgt, wenn die klinische Präsentation und klinisch indizierte EKGs, nicht jedoch eine Schrittmacherabfrage berücksichtigt werden [41]. Inwieweit bei Patienten mit paroxysmalem Vorhofflimmern strukturelle Veränderungen des Vorhofs für das Schlaganfallrisiko von Bedeutung sind, ist aktueller Gegenstand der Forschung [42] und könnte die Diskussion um die Relevanz einer „Mindestdauer“ einer Vorhofflimmerepisode relativieren. 
\ Tab.2 Ausgewählte prospektive Kohortenstudien zur Detektion von Vorhofflimmern nach akutem ischämischem Schlaganfall.

\begin{tabular}{|c|c|c|c|c|c|}
\hline $\begin{array}{l}\text { Studie } \\
\text { [Referenz] }\end{array}$ & $\begin{array}{l}\text { Monitoring- } \\
\text { Verfahren }\end{array}$ & n & $\begin{array}{l}\text { Alter } \\
\text { (Jahre) }\end{array}$ & $\begin{array}{l}\text { Vorhofflimmer- } \\
\text { nachweis }\end{array}$ & Kommentar \\
\hline $\begin{array}{l}\text { FIND AF } \\
{[36]}\end{array}$ & $\begin{array}{l}7 \text { Tage Langzeit- } \\
\text { EKG }\end{array}$ & 224 & $68 \pm 13$ & $\begin{array}{l}4,8 \% \text { nach } 24 h \\
6,3 \% \text { nach } 48 h \\
12,5 \% \text { nach } 7 \text { Tagen }\end{array}$ & $\begin{array}{l}\text { Bei etwa der Hälfte der Schlaganfallpatienten } \\
\text { mit erstmaligem Vorhofflimmern wurde das } \\
\text { Vorhofflimmern erstmalig nach stationärer } \\
\text { Entlassung im Langzeit-EKG nachgewiesen. }\end{array}$ \\
\hline $\begin{array}{l}\text { IDEAS } \\
{[35]}\end{array}$ & $\begin{array}{l}3 \text { Tage Langzeit- } \\
\text { EKG }\end{array}$ & 1135 & $67 \pm 13$ & $\begin{array}{l}2,6 \% \text { nach } 24 h \\
4,3 \% \text { nach } 72 h\end{array}$ & $\begin{array}{l}\text { Ein Vorhofflimmer-Nachweis in den ersten } \\
\text { 24-Stunden der EKG-Ableitung gelang in 2,6\% } \\
\text { aller Patienten mit akutem ischämischem } \\
\text { Schlaganfall. }\end{array}$ \\
\hline $\begin{array}{l}\text { Ritter et al. } \\
{[80]}\end{array}$ & $\begin{array}{l}\text { implantierbarer } \\
\text { Ereignisrekorder }\end{array}$ & 60 & $\begin{array}{l}63 \\
{[\text { IQR 49-72] }}\end{array}$ & $\begin{array}{l}17 \% \text { nach im Mittel } \\
64(1-556) \text { Tagen }\end{array}$ & $\begin{array}{l}\text { Implantation erfolgte im Mittel } 13(10-65) \\
\text { Tage nach akutem ischämischem Schlaganfall, } \\
\text { der bis dato als kryptogen eingestuft wurde, } \\
\text { da ein EKG-Monitoring über insgesamt } 96 \mathrm{~h} \\
\text { kein Vorhofflimmern zeigte. Ein parallel } \\
\text { abgeleitetes EKG über } 7 \text { Tage wies bei einem } \\
\text { Patient ein Vorhofflimmern nach. }\end{array}$ \\
\hline $\begin{array}{l}\text { Poli et al. } \\
\text { [23] }\end{array}$ & $\begin{array}{l}\text { implantierbarer } \\
\text { Ereignisrekorder }\end{array}$ & 75 & $66 \pm 13$ & $\begin{array}{l}28 \% \text { nach } 6 \text { Monaten } \\
33 \% \text { nach } 12 \text { Monaten }\end{array}$ & $\begin{array}{l}\text { Unabhängige Risikofaktoren für die Detektion } \\
\text { von Vorhofflimmern nach bis dato kryptoge- } \\
\text { nem Schlaganfall waren die Einschlusskriterien } \\
\text { „atrial run“ bzw. Vorhofdurchmesser }>45 \mathrm{~mm} \text {. } \\
\text { Weitere Einschlusskriterien waren ein } \\
\mathrm{CHA}_{2} \mathrm{DS}_{2} \text {-VASc } \geq 4 \text {, ein LAA Fluss } \leq 0,2 \mathrm{~m} / \mathrm{s} \\
\text { oder Spontanechokontrast im linken Vorhof. }\end{array}$ \\
\hline
\end{tabular}

\section{Welchen Qualitätsstandards sollte ein EKG-Monitoring unterliegen?}

Bisher liegen wenige Studien zur systematischen Auswertung des EKG-Monitorings auf der Stroke Unit vor [20,21]. Eine im Jahr 2014 unter Schirmherrschaft der DSG durchgeführte Umfrage zur Detektion von Vorhofflimmern [43], an der sich 171 Betreiber einer zertifizierten Stroke Unit beteiligten, verdeutlicht bestehende Möglichkeiten zur Optimierung der stationären EKG-Diagnostik in der täglichen Praxis. Daher halten es die Autoren für wichtig, Standards für eine qualitativ hochwertige „Rhythmusvisite“ festzulegen, die auch eine Einbindung von Kardiologen festlegt. Es sollte sichergestellt werden, dass abgeleitete EKG-Daten zumindest einmal am Tag durch geschultes und erfahrenes Personal standardisiert analysiert werden [21]. Eine automatisierte Analyse ist in diesem Kontext hilfreich, bedarf aber einer „manuellen“ Validierung der EKG-Befunde und einer ärztlichen Diagnosestellung [20,21,44]. Neben der Detektion von Vorhofflimmern und Vorhofflattern müssen dabei auch potenziell lebensbedrohliche Arrhythmien beachtet werden. Die auf der Stroke Unit abgeleiteten EKG-Daten sollten aufgezeichnet werden, um offline systematisch analysiert werden zu können. Die derzeit gebräuchlichen Monitoring-Systeme besitzen in aller Regel eine diesbezügliche Speicherfunktion. Eine „Rhythmusvisite“ kann sich beispielsweise an dem in - Abb. 1 dargestellten Schema orientieren, das im Rahmen einer prospektiven monozentrischen Kohortenstudie zu einer verbesserten Detektion von Vorhofflimmern führte [21].

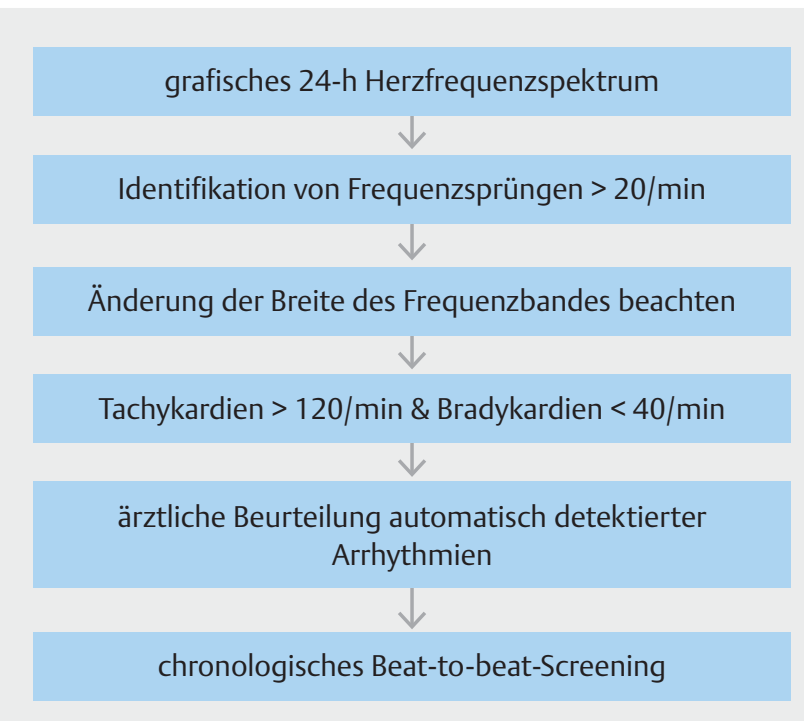

- Abb. 1 Schema zur systematischen Auswertung des EKG-Monitorings auf der Stroke Unit (modifiziert nach [21]). 


\section{Wie lange sollte ein EKG-Monitoring nach ischämischem Schlaganfall mindestens andauern?}

Derzeit gibt es in Deutschland etwas mehr als 300 zertifizierte Stroke Units, in denen mehr als $80 \%$ aller Patienten mit akutem ischämischem Schlaganfall auf hohem Qualitätsniveau behandelt werden. Im Einklang mit den Leitlinien der Deutschen Gesellschaft für Neurologie [25] erfolgt dabei eine ätiologisch orientierte Patientenauswahl sowohl für ein konventionelles Langzeit-EKG als auch für ein erweitertes EKG-Monitoring. Aufgrund struktureller Gegebenheiten und ökonomischer Zwänge ist es den Stroke Units nicht ohne Weiteres möglich, den bisherigen diagnostischen Standard der kardialen Rhythmusdiagnostik auf breiter Basis auszuweiten. Aus Sicht der Autoren und der Stroke Unit-Kommission der Deutschen Schlaganfallgesellschaft sind hierfür entsprechende Strukturanpassungen in den verantwortlichen Fachabteilungen erforderlich.

Es besteht Konsens unter den Autoren, dass ein Basis-EKGMonitoring nach ischämischem Schlaganfall idealerweise für zumindest 72 Stunden erfolgen sollte, um die Wahrscheinlichkeit für den erstmaligen Nachweis von Vorhofflimmern zu erhöhen. Ein Monitoring über 72 Stunden wird auch von den aktuellen Leitlinien der Europäischen Gesellschaft für Kardiologie empfohlen [4], auch wenn dies bisher noch nicht als klinische Routine in der Versorgung von Schlaganfallpatienten anzusehen ist. Dabei erscheint es unerheblich, ob dieses Monitoring in Gänze mittels Telemetrie auf der Stroke Unit oder mittels Holter-EKG erfolgt, sofern eine qualitativ hochwertige, standardisierte Auswertung des EKGs sichergestellt wird [21]. Es sollte kein Unterschied zwischen Patienten mit (gesichertem) ischämischem Schlaganfall und Patienten mit einer TIA gemacht werden, wenn relevante Differenzialdiagnosen einer TIA nicht wahrscheinlich erscheinen. Bei kürzerer stationärer Verweildauer ist für TIA-Patienten eine ambulante Fortsetzung des EKG-Monitorings empfehlenswert, um kumulativ zumindest 72 Stunden zu erreichen. Zudem sollte bei Schlaganfallpatienten ein EKG-Monitoring auch dann durchgeführt werden, wenn eine nicht kardio-embolische Genese des ischämischen Schlaganfalls angenommen wird, da der Nachweis eines Vorhofflimmerns auch für die Sekundärprävention dieser Patienten relevant ist. Selbst bei Vorliegen von absoluten Kontraindikationen für eine dauerhafte orale Antikoagulation ist ein EKG-Monitoring in der Akutphase des ischämischen Schlaganfalls zu empfehlen, da neben dem Auftreten sonstiger relevanter Herzrhythmusstörungen - wie Vorhofflattern, ein AV-Block II ${ }^{\circ}$ oder III', eine ventrikuläre Tachykardie oder passagere Asystolie [45] - bei bestimmten Patienten bei Nachweis eines Vorhofflimmerns ein interventioneller Verschluss des linken Vorhofohrs erwogen werden kann [4]. Dieser Eingriff sollte jedoch idealerweise im Rahmen einer randomisierten klinischen Studie erfolgen.

Für ein EKG-Monitoring über 72 Stunden hinaus müssen der resultierende Kostenaufwand, die mit der Zeit abnehmende Compliance bei nicht-invasiven Monitoring-Verfahren und die Erfolgsaussichten des Monitorings gegeneinander abgewogen werden ( $>$ Tab.3), da ein kontinuierliches EKG-Monitoring von 30 Tagen, wie von den aktuellen US-amerikanischen Leitlinien für Schlaganfallpatienten mit bis dato kryptogenem Schlaganfall empfohlen [28], in der klinischen Praxis nicht regelhaft umsetzbar erscheint. Eine ganze Reihe von klinischen, EKG- bzw. echokardiografischen Parametern sind beschrieben worden ( $\triangleright$ Tab.4), die die Wahrscheinlichkeit erhöhen ein Vorhofflimmern nach einem ischämischen Schlaganfall erstmals zu diagnostizieren. Im Folgenden wird die diesbezügliche Datenlage dargestellt.

\section{Relevanz der zerebralen Bildgebung für die Detektion von Vorhofflimmern}

Bestimmte Läsionsmuster in der zerebralen Bildgebung suggerieren eine kardio-embolische Genese bzw. eine Emboliequelle im Bereich der Abgänge der supraaortalen Arterien, wie beispielsweise multilokuläre territoriale Infarktmuster, die verschiedene zerebrale Gefäßterritorien betreffen [46,47]. Der spätere Nachweis eines Vorhofflimmerns korrelierte in der CRYSTAL-AF Studie ( $\vee$ Tab. 1 ) jedoch nicht mit dem zerebralen Läsionsmuster [48]. Es ist hervorzuheben, dass ein gewisser Anteil der Patienten mit „lakunärem“ Infarktmuster ein Vorhofflimmern aufweist $[12,13]$, sodass ein standardisiertes (Basis-) EKG-Monitoring auch bei Patienten mit „lakunärem“ Infarkt angezeigt ist, auch wenn die Wahrscheinlichkeit eines erstmaligen Nachweises von Vorhofflimmern weniger hoch ist, als bei nicht-lakunären Infarktmustern [49]. Weiterhin zu beachten sind zerebrale Läsionen, die eine autonome Imbalance induzieren und somit das Auftreten von Vorhofflimmern vermitteln könnten [8]. Eine diesbezüglich besonders vulnerable Hirnregion scheint im Bereich der rechten Inselregion zu liegen [9]. Die Frage, ob das nach einem Schlaganfall detektierte Vorhofflimmern Ursache oder Folge des Schlaganfalls ist, mag konsekutiv im Einzelfall nicht zu klären sein. Diese Differenzierung besitzt jedoch derzeit nur akademische Bedeutung, da keine Daten vorliegen, die ein intermittierendes Bestehen von Schlaganfall-induziertem Vorhofflimmern belegen.

KURZGEFASST

Gemäß der aktuellen Datenlage kann anhand der Befunde der zerebralen Bildgebung kein sicherer Rückschluss auf die Schlaganfallätiologie gezogen werden. Der Nachweis (multipler) nicht-lakunär konfigurierter zerebraler Ischämien findet sich häufiger bei Schlaganfallpatienten mit Vorhofflimmern, sodass ein verlängertes EKG-Monitoring bei diesen Schlaganfallpatienten erwogen werden kann ( Tab.4). 
Dab. 3 Möglichkeiten des Herzrhythmus-Monitorings nach Schlaganfall (nach [81, 82]).

\begin{tabular}{|c|c|c|c|}
\hline & Indikation gemäß Leitlinien & praktische Aspekte & Qualitätsstandard \\
\hline 12-Kanal-EKG & $\begin{array}{l}\text { bei stationärer Aufnahme } \\
\text { zur Detektion von Vorhof- } \\
\text { flimmern empfohlen } \\
\text { (Klasse I, Level B) [4]. }\end{array}$ & $\begin{array}{l}\text { Sollte bei Aufnahme, bei Auffälligkei- } \\
\text { ten am EKG-Monitor bzw. auftreten- } \\
\text { den Beschwerden stationär erfolgen. } \\
\text { Ambulante Kontrollen als „Minimal- } \\
\text { variante“ eines Herzrhythmus-Moni- } \\
\text { torings praktikabel. }\end{array}$ & ärztliche Beurteilung \\
\hline Stroke-Unit-Monitoring & $\begin{array}{l}\text { Für Schlaganfallpatienten } \\
\text { wird eine EKG-Ableitung } \\
\text { über } 72 \text { Stunden empfohlen } \\
\text { (Klasse I, Level B) [4]. }\end{array}$ & $\begin{array}{l}\text { Die Differenzierung von atrialen Tachy- } \\
\text { kardien ist problematisch. Die Imple- } \\
\text { mentierung eines automatischen } \\
\text { Detektions-Algorithmus kann sinnvoll } \\
\text { sein, erfordert jedoch eine additive } \\
\text { manuelle Auswertung [21]. Falsch- } \\
\text { positive Alarme sind sehr häufig [83]. }\end{array}$ & $\begin{array}{l}\text { ärztliche Beurteilung } \\
\text { Systematische, regelmäßige } \\
\text { (tägliche) Evaluation anhand eines } \\
\text { standardisierten Auswertungs- } \\
\text { Algorithmus ( } \triangleright \text { Abb. 1) erhöht } \\
\text { die Detektion [21]. }\end{array}$ \\
\hline Langzeit-EKG & $\begin{array}{l}\text { Für Schlaganfallpatienten } \\
\text { wird eine EKG-Ableitung } \\
\text { über } 72 \text { Stunden (Klasse I, } \\
\text { Level B) [4] bzw. über bis zu } \\
30 \text { Tage empfohlen [28]. }\end{array}$ & $\begin{array}{l}\text { Die Aufzeichnung mehrerer EKG- } \\
\text { Kanäle (z. B. 5-poliges Kabel) kann die } \\
\text { Qualität der Aufzeichnung deutlich } \\
\text { erhöhen. }\end{array}$ & $\begin{array}{l}\text { ärztliche Beurteilung } \\
\text { Durch Nutzung eines Corelabs } \\
\text { kann in Studien eine Qualitäts- } \\
\text { steigerung erreicht werden. }\end{array}$ \\
\hline $\begin{array}{l}\text { externe Ereignisrekorder } \\
\text { (getriggerte Aufzeichnung) }\end{array}$ & $\begin{array}{l}\text { derzeit keine explizite } \\
\text { Empfehlung }\end{array}$ & $\begin{array}{l}\text { Hauptvorteil gegenüber Langzeit-EKG } \\
\text { ist die Zeitersparnis bei der Auswertung } \\
\text { durch Vorauswahl auffälliger Episoden } \\
\text { anhand von Detektions-Algorithmen, } \\
\text { die kurze Vorhofflimmer-Episoden } \\
\text { nicht detektieren. Hohe Rate falsch- } \\
\text { positiver Befunde, die eine manuelle } \\
\text { Nachbefundung erfordern und den } \\
\text { Einsatz limitieren. }\end{array}$ & $\begin{array}{l}\text { ärztliche Beurteilung } \\
\text { Eine hohe Compliance des } \\
\text { Patienten ist erforderlich. }\end{array}$ \\
\hline $\begin{array}{l}\text { externe Ereignisrekorder } \\
\text { (temporär appliziert) }\end{array}$ & $\begin{array}{l}\text { derzeit keine explizite } \\
\text { Empfehlung }\end{array}$ & $\begin{array}{l}\text { bequem, längere Überwachungs- } \\
\text { Intervalle möglich. Kurze Vorhofflim- } \\
\text { mer-Episoden werden mit hoher Wahr- } \\
\text { scheinlichkeit nicht detektiert. Hohe } \\
\text { Rate falsch-positiver Befunde, die eine } \\
\text { manuelle Nachbefundung erfordern } \\
\text { und den Einsatz limitieren. }\end{array}$ & $\begin{array}{l}\text { ärztliche Beurteilung } \\
\text { Eine hohe Compliance ist erforder- } \\
\text { lich. EKG-Qualität durch Artefakte } \\
\text { oft schlechter als Holter-EKG. }\end{array}$ \\
\hline $\begin{array}{l}\text { implantierter } \\
\text { Ereignisrekorder }\end{array}$ & $\begin{array}{l}\text { sollte bei Schlaganfall- } \\
\text { patienten erwogen werden } \\
\text { (Klasse Ila, Level B) [4] }\end{array}$ & $\begin{array}{l}\text { Nach erfolgreicher Implantation Über- } \\
\text { wachungs-Intervalle von } 2 \text { - } 4 \text { Jahren. } \\
\text { Kurze Episoden von Vorhofflimmern } \\
\text { werden mit hoher Wahrscheinlichkeit } \\
\text { nicht detektiert bei sonst kontinuier- } \\
\text { licher Detektion mit hoher Sensitivität } \\
\text { und Spezifität. Nachteile sind die Inva- } \\
\text { sivität des Verfahrens sowie die hohen } \\
\text { Kosten. }\end{array}$ & $\begin{array}{l}\text { ärztliche Beurteilung } \\
\text { Ableitung ist unabhängig von der } \\
\text { Compliance. Qualität des EKGs ist } \\
\text { durch artifizielle Überlagerung oft } \\
\text { schlechter als Holter-EKG. }\end{array}$ \\
\hline $\begin{array}{l}\text { implantierte SM/ICD } \\
\text { mit atrialer Sonde }\end{array}$ & $\begin{array}{l}\text { Ein Auslesen des Devices } \\
\text { wird empfohlen (Klasse I, } \\
\text { Level B) [4]. }\end{array}$ & $\begin{array}{l}\text { Sofern vorab nicht geschehen, sollte } \\
\text { die Detektionsfunktion aktiviert und } \\
\text { das Gerät ggf. mit einer höheren Fre- } \\
\text { quenz ausgelesen werden. }\end{array}$ & ärztliche Beurteilung \\
\hline
\end{tabular}

\section{Relevanz der kardialen Bildgebung für die Detektion von Vorhofflimmern}

In der Echokardiografie können strukturelle und funktionelle kardiale und herznahe Veränderungen nachgewiesen werden, die auf ein erhöhtes Risiko für Vorhofflimmern hinweisen. Bestimmte Klappenvitien, insbesondere rheumatisch bedingte Mitralklappenstenosen oder hochgradige Mitral- und Trikuspi- dalklappeninsuffizienzen besitzen eine prädiktive Wertigkeit für den Nachweis von Vorhofflimmern. Die transösophageale Echokardiografie (TOE) bietet eine höhere Auflösung für die Darstellung von Vorhofstrukturen (z.B. Vorhofohrthromben) und der Atheromlast der thorakalen Aorta [50], die von transthorakal oft nicht sicher beurteilt werden können. Als mögliche Folge eines Vorhofflimmerns können in der TOE ein spontaner Echokontrast und solide Thromben im Vorhof nachweisbar sein. Ob die Morphologie des Vorhofohrs mit der Thrombogeni- 
- Tab.4 Konsensus-Empfehlung zur möglichen Relevanz klinischer, bildgebender bzw. elektrokardiografischer Parameter für die erstmalige Detektion von Vorhofflimmern nach ischämischem Schlaganfall.

\begin{tabular}{|l|l|l|}
\hline \multicolumn{2}{|l|}{ Wahrscheinlichkeit für erstmaligen Nachweis von Vorhofflimmern } & \\
\hline & vergleichsweise gering & vergleichsweise hoch \\
\hline Patientenalter & $<60$ Jahre & $\geq 75$ Jahre \\
\hline supraventrikuläre Extrasystolen & $<120$ pro 24 Stunden & $\geq 480$ pro 24 Stunden \\
\hline längster „atrial run“ & $<5$ Schläge & $\geq 20$ Schläge \\
\hline BNP bzw. & $<50 \mathrm{pg} / \mathrm{ml} \mathrm{bzw.}$ & $>100 \mathrm{pg} / \mathrm{ml} \mathrm{bzw.}$ \\
\hline NT-proBNP & $<200 \mathrm{pg} / \mathrm{ml}$ & $>400 \mathrm{pg} / \mathrm{ml}$ \\
\hline linksatrialer Durchmesser & $<40 \mathrm{~mm}$ & $>45 \mathrm{~mm}$ \\
\hline Schlaganfallätiologie & $\begin{array}{l}\text { lakunärer Schlaganfall; } \\
\text { andere definitive Ursache }\end{array}$ & $\begin{array}{l}\text { arterio-arterielle Embolie; kryptogen bzw. } \\
\text { ESUS; kardiale Ursache außer Vorhofflimmern }\end{array}$ \\
\hline
\end{tabular}

tät korreliert, wird aktuell untersucht [51]. Die mögliche Bedeutung der im Gewebedoppler möglichen Darstellung der Verformungsgeschwindigkeit des linken Vorhofs (linksatriales „strain“) für den Nachweis von Vorhofflimmern [52,53] nach ischämischem Schlaganfall muss anhand weiterer Studien belegt werden. Die in der transthorakalen Echokardiografie gemessene Größe des linken Vorhofs ist hingegen als Prädiktor für ein paroxysmales Vorhofflimmern anzusehen [54]. Ebenso scheint der Kombination aus linksatrialem Volumenindex und atrialer Funktion eine prädiktive Wertigkeit für den Nachweis von Vorhofflimmern zuzukommen [55, 56], wobei eine diesbezügliche Validierung in größeren Schlaganfallkohorten erfolgen sollte. Der Stellenwert der Magnetresonanztomografie bzw. Computertomografie, die eine zunehmend bessere räumliche und zeitliche Auflösung zur funktionellen Charakterisierung des Herzens ermöglichen, wird derzeit in Studien untersucht [57].

\section{KURZGEFASST}

Die Größe des linken Vorhofs kann nach gegenwärtiger Datenlage für die kumulative Dauer des EKG-Monitorings nach ischämischem Schlaganfall berücksichtigt werden ( Tab.4). Ein normal großer linker Vorhof schließt jedoch das Bestehen eines Vorhofflimmerns nicht aus.

\section{Relevanz von Biomarkern für die Detektion von Vorhofflimmern}

Aufgrund pathophysiologischer Konzepte zur Entstehung von Vorhofflimmern erscheinen verschiedene Biomarker interessant, die bei einer atrialen Dilatation (natriuretische Peptide), einer Schädigung von Myozyten (Troponin), einer atrialen Fibrose (IL-6, CRP), bei Gerinnungsstörungen (D-Dimer), vaskulärer Schädigung (IL-6, CRP, GFR, Cystatin C, Marker der endothelialen Funktion), einer eingeschränkten kardialen Funktion (natriuretische Peptide) oder einer eingeschränkten Hämodyna- mik (natriuretische Peptide, GFR, Cystatin C) in erhöhten Konzentrationen messbar sein können [9,58,59]. Für die meisten der aufgelisteten Biomarker gibt es bisher noch keine überzeugenden Daten aus großen prospektiven Studien. Als besonders vielversprechend für die Detektion von Vorhofflimmern haben sich bisher das Akute-Phase-Protein CRP und die natriuretischen Peptide NT-proBNP bzw. BNP herausgestellt [60,61]. Für die natriuretischen Peptide ließ sich das auch in Schlaganfallkollektiven bestätigen [62-64]. Trotz einer Erhöhung der natriuretischen Peptide in der Akutphase eines Schlaganfalls kann anhand von teils retrospektiv erhobenen Studiendaten davon ausgegangen werden, dass BNP-Werte von $>100 \mathrm{pg} / \mathrm{ml}$ bzw. NT-proBNP-Werte $>400 \mathrm{pg} / \mathrm{ml}$ bei Schlaganfallpatienten eine prädiktive Wertigkeit für das Auftreten von Vorhofflimmern zukommt [62]. Interessanterweise zeigte eine retrospektive Analyse der WARSS-Studie für Schlaganfallpatienten ohne bekanntes Vorhofflimmern einen Nutzen einer oralen Antikoagulation mittels Warfarin im Vergleich zur Sekundärprävention mittels Acetylsalicylsäure, wenn bei Studieneinschluss ein erhöhtes NT-proBNP bestand [65].

\section{KURZGEFASST}

Patienten mit erhöhtem BNP bzw. NT-proBNP weisen eine höhere Wahrscheinlichkeit für einen Nachweis von Vorhofflimmern auf, was für die kumulative Dauer des EKGMonitorings nach ischämischem Schlaganfall berücksichtigt werden kann. Die in $>$ Tab. 4 vorgeschlagenen cutoffs für BNP bzw. NT-proBNP müssen jedoch noch prospektiv validiert werden. 


\section{Relevanz von EKG-Parametern für die Detektion von Vorhofflimmern}

Eine vermehrte supraventrikuläre Ektopie (excessive supraventricular ectopic activity, ESVEA) bezeichnet eine supraventrikuläre Extrasystolie (innerhalb von 24 Stunden) oder das Bestehen einer supraventrikulären Tachykardie („atrial run“), die weniger als 30 Sekunden andauert. In der Copenhagen Holter Study [66] wiesen Patienten mit ESVEA ein deutlich erhöhtes Risiko für Schlaganfälle und klinisch manifestes Vorhofflimmern auf. Auch Schlaganfallpatienten mit ESVEA zeigen häufiger im Verlauf ein Vorhofflimmern [67]. Allerdings fehlen belastbare Normwerte für die Akutphase des Schlaganfalls. Eine retrospektive Analyse der EMBRACE-Studie ( $\triangleright$ Tab. 1) zeigte zudem, dass der Nachweis von gehäuften atrialen Extrasystolen bei Schlaganfallpatienten mit einer erhöhten Detektionswahrscheinlichkeit von Vorhofflimmern assoziiert war [68].

\section{KURZGEFASST}

Die Suche nach supraventrikulären Extrasystolen und „atrial run“ sollte fester Bestandteil einer Langzeit-EKGAuswertung sein und für die kumulative Dauer des EKGMonitorings berücksichtigt werden ( $>$ Tab. 4). Gemäß aktueller Leitlinien stellt eine vermehrte supraventrikuläre Ektopie jedoch keine Indikation für eine orale Antikoagulation dar.

\section{Relevanz von klinischen Parametern bzw. von Risiko-Skalen für die Detektion von Vorhofflimmern}

Der offenbar stärkste Prädiktor für den Nachweis von Vorhofflimmern ist das Alter ( Tab.4) [69]. Der Einsatz der bisher etablierten Risiko-Skalen für die Vorhersage eines Vorhofflimmerns nach ischämischem Schlaganfall erscheint verzichtbar, da diese Risiko-Skalen im Wesentlichen auf bekannten kardiovaskulären Risikofaktoren basieren und nur teilweise durch weitere diagnostische Parameter ergänzt werden, sodass sich kein wesentlicher Vorteil dieser Risiko-Skalen erkennen lässt.

\section{KURZGEFASST}

Das Patientenalter sollte für die kumulative Dauer des (post-)stationären EKG-Monitorings berücksichtigt werden (siehe $>$ Tab.4). Publizierte Risiko-Skalen für die Detektion von Vorhofflimmern nach ischämischem Schlaganfall oder TIA scheinen im klinischen Alltag gegenwärtig verzichtbar.

\section{Konsensempfehlung zur Stratifikation für ein verlängertes Monitoring anhand von Risikofaktoren}

Im Anschluss an ein Basis-EKG-Monitoring von idealerweise 72 Stunden sollte nach Ansicht der Autoren eine Abschätzung des Vorhofflimmerrisikos anhand der in $>$ Tab. 4 genannten Parameter erwogen werden, um die Dauer verlängerten EKG-Monitorings pragmatisch festzulegen, wie in $\mathbf{A b b} \mathbf{2}$ beispielhaft skizziert. Die Entscheidung über ein verlängertes EKG-Monitoring sollte auf einer schriftlich fixierten Standardprozedur basieren, wie von den aktuellen Zertifizierungskriterien der Stroke Unit-Kommission gefordert [29].

\section{Poststationäres EKG-Monitoring nach ischämischem Schlaganfall}

Ein EKG-Monitoring nach der Akutphase wird von Leitlinien empfohlen $[27,28]$ und erscheint grundsätzlich praktikabel, erfolgt jedoch oft nur in einem begrenzen Maß [7,31,33]. Eine fokussierte und standardisierte Kommunikation zwischen der neurologischen Akutklinik und den ambulanten Behandlern erscheint wichtig für die praktische Umsetzung eines weiteren EKG-Monitorings, wobei systematische Analysen fehlen. Mit welchem technischen Verfahren ein post-stationäres EKG-Monitoring nach ischämischem Schlaganfall oder TIA bestmöglich und kosteneffizient sichergestellt werden kann, ist nicht abschließend geklärt ( Tab.3). Abseits des EKGs steht die Pulspalpitation als etabliertes Verfahren des Rhythmus-Monitorings zur Verfügung, das durch eine Schulung von Angehörigen von Schlaganfallpatienten mit einer vergleichsweise hohen Sensitivität und hoher Spezifität zur Detektion von Vorhofflimmern eingesetzt werden kann [70].

\section{Welche Schlaganfallpatienten sollten ein kontinuierliches EKG-Monitoring erhal- ten?}

Die Berücksichtigung des kardiovaskulären Risikoprofils sowie einzelner EKG- und echokardiografischer Parameter kann bei Schlaganfallpatienten die (post-)stationäre Nachweisrate von Vorhofflimmern mittels eines implantierten Ereignisrekorders erhöhen, wie anhand einer vergleichsweise kleinen monozentrischen Studie gezeigt werden konnte ( $\triangleright$ Tab. 2) [23]. Daher sollten Schlaganfallpatienten, die bestimmte Risikofaktoren für ein Vorhofflimmern aufweisen (siehe $>$ Abb.2) ein verlängertes EKG-Monitoring erhalten. Das nicht-invasive EKG-Monitoring sollte sich an der gegebenen Praktikabilität orientieren und idealerweise mindestens 7 Tage andauern. Da die Compliance für ein noch längeres nicht-invasives Monitoring abnimmt [32], empfehlen die Autoren nach mehrheitlicher Auffassung den Einsatz von implantierbaren Ereignisrekordern bei Schlaganfallpatienten zu erwägen, bei denen ein verlängertes nichtinvasives EKG-Monitoring kein Vorhofflimmern nachgewiesen 


\section{orale Antikoagulation (bei fehlender Kontraindikation)}

$\uparrow$

erstmaliger Nachweis von Vorhofflimmern



EKG-Monitoring über bis zu

72 Stunden

stationäre Telemetrie/

stationäres oder

poststationäres

Langzeit-EKG

Risikostratifizierung in Anlehnung an Tab. 4

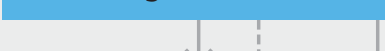

additives nicht-invasives EKG-Monitoring kein weiteres EKG-Monitoring (sofern asymptomatisch) hat, jedoch eine erhöhte Wahrscheinlichkeit für das Bestehen von Vorhofflimmern besteht ( $>$ Tab.4).

\section{Ist ein verlängertes EKG-Monitoring nach kryptogenem Schlaganfall verzichtbar?}

Derzeit wird die Frage untersucht, ob Patienten mit einem mutmaßlich embolischen ischämischen Schlaganfall anstelle einer leitliniengerechten Sekundärprävention mittels Acetylsalicylsäure [26] nicht doch oral antikoaguliert werden sollten. Zuvor konnten zwei randomisierte Studien keinen Vorteil des Vitamin K-Antagonisten Warfarin gegenüber Acetylsalicylsäure nachweisen [71,72]. Nach Veröffentlichung einer operationalisierten Definition, dem „Embolic Stroke of Undetermined Source“ (ESUS) [73], werden derzeit zwei multizentrische randomisierte Studien durchgeführt, in die zusammen mehr als 13000 ESUSPatienten eingeschlossen werden sollen (RESPECT-ESUS, Dabigatran vs. Acetylsalicylsäure [74]; NAVIGATE-ESUS, Rivaroxaban vs. Acetylsalicylsäure, [75]). Für einen Studieneinschluss gefordert wird unter anderem ein zumindest über 24 Stunden anhaltendes (ansonsten aber nicht näher spezifiziertes) EKGMonitoring. Interessant ist insbesondere, ob auch ESUS-Patienten von einer oralen Antikoagulation profitieren, die auch im Studienverlauf kein paroxysmales Vorhofflimmern aufweisen. Um dies besser differenzieren zu können, rekrutiert in Deutschland eine multizentrische randomisierte Studie zum Einsatz von
Apixaban vs. Acetylsalicylsäure (ATTICUS), deren primärer Endpunkt der MRT-basierte Nachweis zerebraler Ischämien bei 500 ESUS-Patienten mit zusätzlichen Risikofaktoren ist. In dieser Studie sollen Schlaganfallpatienten ein kontinuierliches Rhythmusmonitoring erhalten, beispielsweise mit einem implantierbaren Ereignisrekorder [76].

\section{Finanzierung eines verlängerten EKG-Monitorings}

Ein verlängertes stationäres EKG-Monitoring erscheint für bestimmte Schlaganfallpatienten wünschenswert ( $\triangleright$ Tab.4), wird aber - abgesehen vom OPS Code - im DRG-System nicht im Detail abgebildet. Ein relevantes sozioökonomisches Problem stellt derzeit die Finanzierung des poststationären EKG-Monitorings dar, da die Kosten für nicht-invasive EKG-Überwachung bislang von den Krankenkassen offenbar nicht in ausreichendem Maß gegenfinanziert werden. Ein verlängertes Monitoring erscheint eine kosteneffektive Maßnahme zu sein, auch wenn diesbezügliche Angaben variieren. So kostete ein gewonnenes Qualitäts-assoziiertes Lebensjahr unter Verwendung eines 30Tage-EKG-Monitorings in den USA ca. 2000 US\$ [77], bei Verwendung eines 7-Tage-Langzeit-EKGs in Deutschland ca. $3900 €$ [78] und bei Verwendung eines implantierten Ereignisrekorders im Rahmen der Crystal-AF Studie ( $\$$ Tab. 1) $13296 £$ [79]. Die Implantation von Eventrekordern bei Schlaganfallpatienten ist 
bisher im Rahmen des stationären Aufenthalts durch eine entsprechende Erhöhung der DRG gegenfinanziert. Allerdings wird eine ambulant erfolgende Implantation nicht immer refinanziert. Ebenso nicht erstattet wird die langjährige ambulante Analyse der aufgezeichneten EKG-Daten. Nach mehrheitlicher Ansicht der Autoren läge es an den Kostenträgern eine optimierte poststationäre Betreuung von Schlaganfallpatienten zu refinanzieren. Die Wertigkeit eines Disease-Management-Programms sollte für diese Patientengruppe erneut evaluiert werden, auch wenn anzumerken ist, dass anhand einer randomisierten Studie noch zu belegen ist, dass durch ein standardisiertes und verlängertes EKG-Monitoring auch eine Reduktion erneuter Schlaganfälle erreicht werden kann.

\section{ZUSAMMENFASSUNG}

Im Rahmen der diagnostischen Abklärung nach einem ischämischen Schlaganfall ist eine zeitnahe EKG-Diagnostik zum Nachweis eines bis dato nicht entdeckten Vorhofflimmerns unerlässlich. Durch ein verlängertes und qualitativ hochwertiges EKG-Monitoring sowie eine Patientenselektion anhand klinischer, laborchemischer sowie echound elektrokardiografischer Parameter kann die Wahrscheinlichkeit eines erstmaligen Nachweises eines nichtpermanenten Vorhofflimmerns und somit auch die Effizienz des EKG-Monitorings gesteigert werden.

\section{Interessenkonflikt}

K. G. Häusler erhielt Vortragshonorare von Bayer HealthCare, SanofiAventis, Pfizer, Bristol-Myers Squibb, Boehringer Ingelheim, Daiichi Sankyo, Medtronic; Honorare für eine Beratertätigkeit von Edwards Lifesciences, Bayer HealthCare, Pfizer, EIP Pharma sowie Forschungsförderung von Bayer HealthCare und Sanofi-Aventis.

K. Gröschel erhielt Vortragshonorare, Honorare für eine Beratertätigkeit oder Reisekostenunterstützung von Bayer HealthCare, Boehringer Ingelheim, Bristol-Myers Squibb, Daiichi Sankyo und Pfizer.

M. Köhrmann erhielt Vortragshonorare und Honorare für eine Beratertätigkeit von Bayer HealthCare, Pfizer, Bristol-Myers Squibb und Boehringer Ingelheim.

R. B. Schnabel erhielt Vortragshonorare von Sanofi-Aventis und Bristol-Myers Squibb.

S. D. Anker erhielt Honorare von Bayer, Boehringer Ingelheim, Novartis, Servier und Vifor Int. und Forschungsförderung von Abbott Vascular und Vifor Int.

M. Böhm erhielt Forschungsunterstützung und Vortragshonorare von Boehringer Ingelheim, Medtronic, St. Jude, Servier, AstraZeneca und Vifor Pharma.

J. Brachmann erhielt Vortragshonorare, Honorare für Beratertätigkeit oder Reisekostenunterstützung von Bayer HealthCare, Daiichi Sankyo, Boehringer Ingelheim, Novartis, Sanofi-Aventis, Pfizer, BristolMyers Squibb, Medtronic, Biotronik.

H.-C. Diener hat Honorare für Teilnahme an klinischen Studien, Mitarbeit in advisory boards und Vorträge erhalten von: Abbott, Allergan, AstraZeneca, Bayer Vital, Bristol-Meyers-Squibb, Boehringer Ingelheim, BrainsGate, CoAxia, Corimmun, Covidien, Daiichi-Sankyo, DPharm, Fresenius, GlaxoSmithKline, Janssen Cilag, Lilly, Lundbeck, Medtronic, MSD, MindFrame, Neurobiological Technologies, Novar- tis, Novo-Nordisk, Paion, Parke-Davis, Pfizer, Sanofi-Aventis, Schering, Servier, Solvay, St-Jude, Syngis, Tacrelis, Thrombogenics, Wyeth und Yamanouchi. Die Einnahmen wurden an das Universitätsklinikum Essen abgeführt zur Finanzierung von Forschungsstellen im Kopfschmerzzentrum, im Schwindelzentrum und in der Klinik für Neurologie. Forschungsprojekte der Universitätsklinik für Neurologie in Essen wurden unterstützt von: AstraZeneca, GSK, Boehringer Ingelheim, Novartis, Janssen-Cilag und SanofiAventis. Die Universitätsklinik für Neurologie hat Forschungsmittel von den folgenden Institutionen erhalten: DFG, BMBF, EU, NIH, EAST-AFnet, Bertelsmann Stiftung und Heinz-Nixdorf Stiftung.

H.-C. Diener besitzt keine Aktien oder Anteile von Pharmafirmen oder Medizintechnikfirmen. HCD war beteiligt an der Erstellung von Leitlinien der DGN, der DSG, der ESC und EHRA.

W. Doehner erhielt Honorare für Vorträge und Beratertätigkeit von Aimediq, Amgen, Bayer, Boehringer Ingelheim, Sanofi, Sphingotec, Stealth Peptides, Vifor Pharma und war als LKP für Studien von Vifor Pharma und ZS Pharma tätig.

M. Endres erhielt Forschungsförderung von Bayer und Roche sowie Honorarzahlung an die Charité von Amgen, Bayer, BMS, Boehringer Ingelheim, Ever, GSK, MSD, Novartis, Pfizer und Sanofi.

C. Gerloff hat Forschungsgelder für Schlaganfallstudien erhalten von der DFG und der EU sowie Honorare für Beratungstätigkeit oder Vorträge erhalten von Bayer Vital, Boehringer Ingelheim, GlaxoSmithKline, Pfizer, Bristol-Myers Squibb, Sanofi Aventis, Amgen und Prediction BioSciences.

H. B. Huttner erhielt Vortragshonorare, Honorare für eine Beratertätigkeit, Reisekostenunterstützung sowie Forschungsfinanzierungen von Bayer HealthCare, Boehringer-Ingelheim, Novartis und Medtronic.

C. H. Nolte erhielt Honorare für Vortrags- oder Beratungstätigkeit und/oder Reiseunterstützung von Boehringer Ingelheim, Bayer HealthCare, Sanofi-Aventis, Pfizer, Bristol-Myers Squibb und Gore.

M. Kaps erhielt Honorare für Vorträge und Beratungen von Bayer, Boehringer Ingelheim, Daiichi Sankyo.

D. G. Nabavi erhielt Vortragshonorare von Bayer HealthCare, SanofiAventis, Pfizer, Bristol-Myers Squibb, Boehringer Ingelheim, Medtronic; Honorare für eine Beratertätigkeit und Reisekostenunterstützung von AstraZeneca, Bayer HealthCare, Bristol-Myers Squibb und Pfizer.

P. Kirchhof erhielt Forschungsunterstützung von der Europäischen Union, British Heart Foundation, Leducq Foundation, Medical Research Council (UK), DZHK und verschiedenen Pharmafirmen.

W. Pfeilschifter erhielt Beraterhonorar und Reisekosten von Sanofi Aventis, Forschungsförderung, Referentenhonorar und Reisekosten von Boehringer Ingelheim, Reisekosten von Bayer, Referentenhonorar, Forschungsförderung und Reisekosten von Stryker Neurovascular, Forschungsförderung von Novartis Pharma.

B. Pieske erhielt Honorare für Beratertätigkeit und Mitarbeit in Studienkommittees von Bayer Healthcare, MSD, Novartis. Stealth Peptides, Astra-Zeneca, Menarini, und BMS.

S. Poli erhielt Vortragshonorare, Honorare für eine Beratertätigkeit oder Reiseunterstützung von Bayer, Boehringer-Ingelheim, BristolMyers Squibb, Pfizer, Daiichi Sankyo und Werfen sowie Forschungsunterstützung durch Bristol-Myers Squibb, Pfizer, Boehringer-Ingelheim, Daiichi Sankyo und Helena Laboratories.

W. R. Schäbitz erhielt Vortragshonorare, Honorare für Beratertätigkeit oder Reisekostenunterstützung von Bayer HealthCare, Daiichi Sankyo, Boehringer Ingelheim, Sanofi-Aventis, Pfizer, Bristol-Myers Squibb und Medtronic.

T. Steiner erhielt Vortragshonorare von Bayer HealthCare, BristolMyers Squibb, Pfizer, Boehringer Ingelheim, Daiichy Sankyo und Beraterhonorare von Bayer, Bristol-Myers Squibb, Pfizer, Boehringer Ingelheim, Daiichy Sankyo und Medtronic.

G. Thomalla erhielt Honorare als Berater, für Teilnahme an Advisory Board Meetings, oder für Vorträge von Acandis, Bristol-Myers Squibb/Pfizer, Boehringer Ingelheim, Daichii Sankyo, GlaxoSmithKline und Stryker sowie Forschungsförderung von Bayer Vital. 
R. Veltkamp erhielt Honorare für Vorträge und Beratungen von Bayer, Boehringer Ingelheim, Daiichi Sankyo, BMS, Pfizer, Medtronic, ApoplexMedical Technologiees, Biogen, Amgen, Morphosys, Sanofi, AZT Therapeutics, Portola sowie Forschungsunterstützung durch Bayer, Boehringer Ingelheim, BMS, Pfizer, Daiicihi Sankyo, Biogen, ApoplexMedical Technologies.

U. Laufs erhielt Honorare für Beratungs- und Vortragstätigkeit von Amgen, Bayer, Berlin-Chemie, Boehringer-Ingelheim, Daiichi-Sankyo, MSD, Sanofi und Servier.

J. Röther erhielt Honorare für Vorträge und/oder Beratungstätigkeiten von Sanofi-Aventis, Pfizer, Lundbeck, Boehringer Ingelheim, BMS, Bayer Vital, Servier sowie der Deutschen Allgemeinen Krankenversicherung (DAK) und war als LKP für Studien der Firmen Astra Zeneca und Servier tätig.

R. Wachter hat für Beratungstätigkeit, Vorträge oder die Betreuung von Patienten in klinischen Studien Honorare von folgenden Firmen erhalten: Bayer, Berlin Chemie, Boehringer Ingelheim, Bristol-Myers Squibb, CVRx, Johnson\&Johnson, Medtronic, Novartis, Pfizer, Sanofi, Servier.

\section{Literatur}

[1] Heuschmann PU, Buss O, Wagner M et al. Schlaganfallhäufigkeit und Versorgung von Schlaganfallpatienten in Deutschland. Akt Neurol 2010; 37: $333-340$

[2] Statistisches Bundesamt. Gesundheit-Todesursachen in Deutschland 2014. Fachserie 12 Reihe 4 Wiesbaden 2016; www.destatis.de/DE/ Publikationen/Thematisch/ Gesundheit/Todesursachen/Todesursachen2120400147004.pdf

[3] Schnabel RB, Johannsen SS, Wild PS et al. Prevalence and risk factors of atrial fibrillation in Germany: data from the Gutenberg Health Study. Herz 2015; 40: 8-15

[4] Kirchhof P, Benussi S, Kotecha D et al. 2016 ESC Guidelines for the management of atrial fibrillation. Eur Heart J 2016; 37: 2893-2962

[5] Marini C, De Santis F, Sacco S et al. Contribution of atrial fibrillation to incidence and outcome of ischemic stroke: results from a populationbased study. Stroke 2005; 36: 1115-1119

[6] Brachmann J, Morillo CA, Sanna T et al. Uncovering atrial fibrillation beyond short-term monitoring in cryptogenic stroke patients: threeyear results from the cryptogenic stroke and underlying atrial fibrillation trial. Circ Arrhythm Electrophysiol 2016; 9: e003333

[7] Lip GY, Hunter TD, Quiroz ME et al. Atrial fibrillation diagnosis timing, ambulatory ECG monitoring utilization, and risk of recurrent stroke. Circ Cardiovasc Qual Outcomes 2017. doi:10.1161/CIRCOUTCOMES.116.002864

[8] Sposato LA, Riccio PM, Hachinski V. Poststroke atrial fibrillation: cause or consequence? Critical review of current views. Neurology 2014; 82: $1180-1186$

[9] Scheitz JF, Erdur H, Haeusler KG et al. Insular cortex lesions, cardiac troponin, and detection of previously unknown atrial fibrillation in acute ischemic stroke: insights from the troponin elevation in acute ischemic stroke study. Stroke 2015; 46: 1196-1201

[10] Higgins P, Dawson J, MacFarlane PW et al. Predictive value of newly detected atrial fibrillation paroxysms in patients with acute ischemic stroke, for atrial fibrillation after 90 days. Stroke 2014; 45: 2134 2136

[11] Herm J, Konieczny M, Jungehulsing G] et al. Should transesophageal echocardiography be performed in acute stroke patients with atrial fibrillation? J Clin Neurosci 2013; 20: 554-559

[12] Kamel H, Okin PM, Elkind MS et al. Atrial fibrillation and mechanisms of stroke: time for a new model. Stroke 2016; 47: 895 - 900
[13] Demeestere J, Fieuws S, Lansberg MG et al. Detection of atrial fibrillation among patients with stroke due to large or small vessel disease: a meta-analysis. J Am Heart Assoc 2016; 5: e004151

[14] Hart RG. Antithrombotic therapy to prevent stroke in patients who have nonvalvular atrial fibrillation. Ann Intern Med 2007; 146: 857

[15] Ruff CT, Giugliano RP, Braunwald E et al. Comparison of the efficacy and safety of new oral anticoagulants with warfarin in patients with atrial fibrillation: a meta-analysis of randomised trials. Lancet 2014; 383: $955-962$

[16] Ottosen TP, Svendsen ML, Hansen ML et al. Preadmission oral anticoagulant therapy and clinical outcome in patients hospitalised with acute stroke and atrial fibrillation. Dan Med J 2014; 61: A4904

[17] Hellwig S, Grittner U, Audebert H et al. Non-vitamin K-dependent oral anticoagulants have a positive impact on ischaemic stroke severity in patients with atrial fibrillation. Europace 2017. doi:10.1093/europace/eux087 [Epub ahead of print]

[18] Dussault C, Toeg H, Nathan M et al. Electrocardiographic monitoring for detecting atrial fibrillation after ischemic stroke or transient ischemic attack: systematic review and meta-analysis. Circ Arrhythm Electrophysiol 2015; 8: 263-269

[19] Sposato LA, Cipriano LE, Saposnik G et al. Diagnosis of atrial fibrillation after stroke and transient ischaemic attack: a systematic review and meta-analysis. Lancet Neurol 2015; 14: 377-387

[20] Rizos T, Güntner ], Jenetzky E et al. Continuous stroke unit electrocardiographic monitoring versus 24-hour holter electrocardiography for detection of paroxysmal atrial fibrillation after stroke. Stroke 2012; 43: 2689-2694

[21] Kallmünzer B, Breuer L, Hering $C$ et al. A structured reading algorithm improves telemetric detection of atrial fibrillation after acute ischemic stroke. Stroke 2012; 43: 994 - 999

[22] Kishore A, Vail A, Majid A et al. Detection of atrial fibrillation after ischemic stroke or transient ischemic attack: a systematic review and meta-analysis. Stroke 2014; 45: 520 - 526

[23] Poli S, Diedler J, Härtig F et al. Insertable cardiac monitors after cryptogenic stroke - a risk factor based approach to enhance the detection rate for paroxysmal atrial fibrillation. Eur J Neurol 2016; 23 : 375 381

[24] Laufs U, Hoppe UC, Rosenkranz S et al. Cardiac workup after cerebral ischemia. Nervenarzt 2010; 81: 444-462

[25] Deutsche Gesellschaft für Neurologie. Leitlinie „Akuttherapie des ischämischen Schlaganfalls“. 2012; http://www.dgn.org/leitlinien/ 2310-II-22-2012-akuttherapie-des-ischaemischen-schlaganfalls

[26] Deutsche Schlaganfall-Gesellschaft (DSG), Deutsche Gesellschaft für Neurologie (DGN). S3-Leitlinie: Sekundärprophylaxe ischämischer Schlaganfall und transitorische ischämische Attacke - Teil 1. Version 1.0. 31.01. 2015; http://www.awmf.org/leitlinien/detail/II/030-133. html

[27] The European Stroke Organisation (ESO). Guidelines for management of ischaemic stroke and transient ischaemic attack. Cerebrovasc Dis 2008; 25: $457-507$

[28] Kernan WN, Ovbiagele B, Black HR et al. Guidelines for the prevention of stroke in patients with stroke and transient ischemic attack: a guideline for healthcare professionals from the American Heart Association/American Stroke Association. Stroke 2014; 45: 2160 2236

[29] Nabavi DG, Ossenbrink M, Schinkel M et al. Revised certification criteria for regional and national stroke units in Germany. Nervenarzt 2015; 86: 978-988

[30] Higgins P, MacFarlane PW, Dawson J et al. Noninvasive cardiac event monitoring to detect atrial fibrillation after ischemic stroke: a randomized, controlled trial. Stroke 2013; 44: 2525-2531

[31] Sanna T, Diener H, Passman RS et al. Cryptogenic stroke and underlying atrial fibrillation. N Engl J Med 2014; 370: 2478-2486 
[32] Gladstone DJ, Sharma M, Spence JD et al. Cryptogenic stroke and atrial fibrillation. N Engl J Med 2014; 371: 1260

[33] Wachter R, Gröschel K, Gelbrich G et al. Holter-electrocardiogrammonitoring in patients with acute ischaemic stroke (Find-AFRANDOMISED): an open-label randomised controlled trial. Lancet Neurol 2017; 16: $282-290$

[34] Haeusler KG, Kirchhof P, Heuschmann PU et al. Impact of standardized MONitoring for Detection of Atrial Fibrillation in Ischemic Stroke (MonDAFIS): Rationale and design of a prospective randomized multicenter study. Am Heart J 2016; 172: 19-25

[35] Grond M, Jauss M, Hamann G et al. Improved detection of silent atrial fibrillation using 72-hour Holter ECG in patients with ischemic stroke: a prospective multicenter cohort study. Stroke 2013; 44: 3357-3364

[36] Stahrenberg R, Weber-Krüger M, Seegers J et al. Enhanced detection of paroxysmal atrial fibrillation by early and prolonged continuous holter monitoring in patients with cerebral ischemia presenting in sinus rhythm. Stroke 2010; 41: 2884-2888

[37] Boriani G, Glotzer TV, Santini M et al. Device-detected atrial fibrillation and risk for stroke: an analysis of $>10,000$ patients from the SOS AF project (Stroke preventiOn Strategies based on Atrial Fibrillation information from implanted devices). Eur Heart J 2014; 35: 508 - 516

[38] Turakhia MP, Ziegler PD, Schmitt SK et al. Atrial fibrillation burden and short-term risk of stroke: case-crossover analysis of continuously recorded heart rhythm from cardiac electronic implanted devices. Circ Arrhythm Electrophysiol 2015; 8: 1040 - 1047

[39] Ganesan AN, Chew DP, Hartshorne T et al. The impact of atrial fibrillation type on the risk of thromboembolism, mortality, and bleeding: a systematic review and meta-analysis. Eur Heart J 2016; 37: 1591 1602

[40] Van Gelder IC, Healey JS, Crijns HJ et al. Duration of device-detected subclinical atrial fibrillation and occurrence of stroke in ASSERT. Eur Heart J 2017; 38: 1339-1344

[41] Charitos El, Pürerfellner H, Glotzer TV et al. Clinical classifications of atrial fibrillation poorly reflect its temporal persistence: insights from 1,195 patients continuously monitored with implantable devices. J Am Coll Cardiol 2014; 63: 2840-2848

[42] Kamel H, Okin PM, Longstreth WT et al. Atrial cardiopathy: a broadened concept of left atrial thromboembolism beyond atrial fibrillation. Futur Cardiol 2015; 11: $323-331$

[43] Rizos T, Quilitzsch A, Busse O et al. Diagnostic work-up for detection of paroxysmal atrial fibrillation after acute ischemic stroke: crosssectional survey on german stroke units. Stroke 2015; 46: $1693-$ 1695

[44] Uphaus T, Grings A, Gröschel S et al. Automatic detection of paroxysmal atrial fibrillation in patients with ischaemic stroke: better than routine diagnostic workup? Eur J Neurol 2017; 24: 990 - 994

[45] Kallmünzer B, Breuer L, Kahl N et al. Serious cardiac arrhythmias after stroke: incidence, time course, and predictors - a systematic, prospective analysis. Stroke 2012; 43: $2892-2897$

[46] Miller DJ, Khan MA, Schultz LR et al. Outpatient cardiac telemetry detects a high rate of atrial fibrillation in cryptogenic stroke. J Neurol Sci 2013; 324: 57-61

[47] Bhatt A, Majid A, Razak A et al. Predictors of occult paroxysmal atrial fibrillation in cryptogenic strokes detected by long-term noninvasive cardiac monitoring. Stroke Res Treat 2011; 2011: 172074

[48] Bernstein RA, Di Lazzaro V, Rymer MM et al. Infarct topography and detection of atrial fibrillation in cryptogenic stroke: results from CRYSTAL AF. Cerebrovasc Dis 2015; 40: 91 - 96

[49] Gaillard N, Deltour S, Vilotijevic B et al. Detection of paroxysmal atrial fibrillation with transtelephonic EKG in TIA or stroke patients. Neurology 2010; 74: 1666 - 1670

[50] Nakanishi K, Homma S. Role of echocardiography in patients with stroke. J Cardiol 2016; 68: 91 - 99
[51] Lupercio F, Carlos RJ, Briceno DF et al. Left atrial appendage morphology assessment for risk stratification of embolic stroke in patients with atrial fibrillation: a meta-analysis. Heart Rhythm 2016; 13: $1402-1409$

[52] Kim D, Shim CY, Cho IJ et al. Incremental value of left atrial global longitudinal strain for prediction of post stroke atrial fibrillation in patients with acute ischemic stroke. J Cardiovasc Ultrasound 2016; 24: $20-27$

[53] Sarvari SI, Haugaa KH, Stokke TM et al. Strain echocardiographic assessment of left atrial function predicts recurrence of atrial fibrillation. Eur Heart J Cardiovasc Imaging 2016; 17: 660 -667

[54] Broughton ST, O'Neal WT, Salahuddin T et al. The influence of left atrial enlargement on the relationship between atrial fibrillation and stroke. J Stroke Cerebrovasc Dis 2016; 25: 1396 - 1402

[55] Stahrenberg R, Edelmann F, Haase B et al. Transthoracic echocardiography to rule out paroxysmal atrial fibrillation as a cause of stroke or transient ischemic attack. Stroke 2011; 42: 3643-3645

[56] Waldenhjort D, Sobocinski Doliwa P et al. Echocardiographic measures of atrial function may predict atrial fibrillation in stroke patients. Scand Cardiovasc J 2016; 50: 236-242

[57] Haeusler KG, Grittner U, Fiebach JB et al. HEart and BRain interfaces in Acute ischemic Stroke (HEBRAS) - rationale and design of a prospective oberservational cohort study. BMC Neurol 2015; 15: 213

[58] Hijazi Z, Oldgren J, Siegbahn A et al. Biomarkers in atrial fibrillation: a clinical review. Eur Heart J 2013; 34: 1475-1480

[59] Camm AJ, Savelieva I, Potpara T et al. The changing circumstance of atrial fibrillation - progress towards precision medicine. J Intern Med 2016; 279: $412-427$

[60] Schnabel RB, Larson MG, Yamamoto JF et al. Relations of biomarkers of distinct pathophysiological pathways and atrial fibrillation incidence in the community. Circulation 2010; 121: 200-207

[61] Sinner MF, Stepas KA, Moser CB et al. B-type natriuretic peptide and $\mathrm{C}$-reactive protein in the prediction of atrial fibrillation risk: the CHARGE-AF Consortium of community-based cohort studies. Europace 2014; 16: $1426-1433$

[62] Wachter R, Lahno R, Haase B et al. Natriuretic peptides for the detection of paroxysmal atrial fibrillation in patients with cerebral ischemia - the Find-AF study. PLoS One 2012; 7: e34351

[63] Llombart V, Antolin-Fontes A, Bustamante A et al. B-type natriuretic peptides help in cardioembolic stroke diagnosis: pooled data metaanalysis. Stroke 2015; 46: 1187-1195

[64] Rodriguez-Yanez M, Arias-Rivas S, Santamaria-Cadavid M et al. High pro-BNP levels predict the occurrence of atrial fibrillation after cryptogenic stroke. Neurology 2013; 81: 444-447

[65] Longstreth WTJr, Kronmal RA, Thompson JL et al. Amino terminal proB-type natriuretic peptide, secondary stroke prevention, and choice of antithrombotic therapy. Stroke 2013; 44: 714-719

[66] Binici Z, Intzilakis T, Nielsen OW et al. Excessive supraventricular ectopic activity and increased risk of atrial fibrillation and stroke. Circulation 2010; 121: $1904-1911$

[67] Weber-Krüger M, Gröschel K, Mende M et al. Excessive supraventricular ectopic activity is indicative of paroxysmal atrial fibrillation in patients with cerebral ischemia. PLoS One 2013; 8: e67602

[68] Gladstone DJ, Dorian P, Spring M et al. Atrial premature beats predict atrial fibrillation in cryptogenic stroke: results from the EMBRACE trial. Stroke 2015; 46: 936 - 941

[69] Thijs VN, Brachmann J, Morillo CA et al. Predictors for atrial fibrillation detection after cryptogenic stroke: Results from CRYSTAL AF. Neurology 2016; 86: $261-269$

[70] Kallmünzer B, Bobinger $\mathrm{T}$, Kahl $\mathrm{N}$ et al. Peripheral pulse measurement after ischemic stroke: A feasibility study. Neurology 2014; 83: 598 603 
[71] ESPRIT study group. Medium intensity oral anticoagulants versus aspirin after cerebral ischaemia of arterial origin (ESPRIT): a randomised controlled trial. Lancet Neurol 2007; 6: 115-124

[72] Mohr JP, Thompson JL, Lazar RM et al. A comparison of warfarin and aspirin for the prevention of recurrent ischemic stroke. N Engl J Med 2001; 345: $1444-1451$

[73] Hart RG, Diener HC, Coutts SB et al. Embolic strokes of undetermined source: the case for a new clinical construct. Lancet Neurol 2014; 13 : $429-438$

[74] Diener HC, Easton JD, Granger CB et al. Design of Randomized, double-blind, Evaluation in secondary Stroke Prevention comparing the EfficaCy and safety of the oral Thrombin inhibitor dabigatran etexilate vs. acetylsalicylic acid in patients with Embolic Stroke of Undetermined Source (RE-SPECT ESUS). Int J Stroke 2015; 10: 1309-1312

[75] Hart RG, Sharma M, Mundl H et al. Rivaroxaban for secondary stroke prevention in patients with embolic strokes of undetermined source: design of the NAVIGATE ESUS randomized trial. Eur Stroke J 2016; 1: $146-154$

[76] Geisler T, Poli S, Meisner C et al. Apixaban for treatment of embolic stroke of undetermined source (ATTICUS randomized trial): rationale and study design. Int J Stroke; 2016 [Epub ahead of print]
[77] Yong JH, Thavorn K, Hoch JS et al. Potential cost-effectiveness of ambulatory cardiac rhythm monitoring after cryptogenic stroke. Stroke 2016; 47: $2380-2385$

[78] Mayer F, Stahrenberg R, Gröschel K et al. Cost-effectiveness of 7-dayHolter monitoring alone or in combination with transthoracic echocardiography in patients with cerebral ischemia. Clin Res Cardiol 2013; 102: $875-884$

[79] Diamantopoulos A, Sawyer LM, Lip GY et al. Cost-effectiveness of an insertable cardiac monitor to detect atrial fibrillation in patients with cryptogenic stroke. Int J Stroke 2016; 11: $302-312$

[80] Ritter MA, Kochhäuser S, Duning T et al. Occult atrial fibrillation in cryptogenic stroke: detection by 7-day electrocardiogram versus implantable cardiac monitors. Stroke 2013; 44: 1449-1452

[81] Wachter R. Rhythmuskontrolle nach Apoplex - Wie lange? Kompendium Herz-Kreislauf Medizin 2016; 12: 30-34

[82] Mittal S, Movsowitz C, Steinberg JS. Ambulatory external electrocardiographic monitoring: focus on atrial fibrillation. J Am Coll Cardiol 2011; 58: $1741-1749$

[83] Kurka N, Bobinger T, Kallmünzer B et al. Reliability and limitations of automated arrhythmia detection in telemetric monitoring after stroke. Stroke 2015; 46: 560 - 563 